\title{
EVALUATION OF THE SEISMIC RESPONSE OF MASONRY BULDINGS BASED ON ENERGY FUNCTIONS
}

\author{
D. Benedetti ${ }^{1}$, P. Carydis ${ }^{2}$, M.P. Limongelli ${ }^{1}$ \\ ${ }^{1}$ Department of Structural Engineering, Politecnico di Milano \\ ${ }^{2}$ Laboratory of Earthquake Engineering, National Technical University of Athens
}

\begin{abstract}
SUMMARY
This work is based on energies evaluated from the responses of 12 stone and brick masonry systems subjected to 58 shaking table tests. The evolution of input energy during a damaging base excitation is correlated to the change of the damage patterns of the considered buildings. The comparison among energies dissipated and adsorbed by the buildings during the various shocks acting on them gives some hints on strengthening strategies. It is found that damage to spandrel beams produces a more significant energy absorption than other types of damage.
\end{abstract}

Keywords: energy functions, damage index, masonry buildings, strengthening

\section{INTRODUCTION}

Few years ago a large number of shaking table tests on masonry buildings has been carried out in the realm of an experimental research funded by the European Commission (CEC). The aim of the research, whose main results can be found in ref.1, was to analyze the efficiency of various retrofitting techniques in improving the seismic behavior of non-engineered masonry buildings. Buildings were scaled 1:2 and were of two types: stone and brick masonry. The latters were tested in their original 
configurations up to medium-severe damage and then repaired and again tested. Stone masonry systems were strengthened previously to tests. The only one stone masonry building tested in its original configuration collapsed due to wall separation. Buildings were excited by three-component base motions of similar peak acceleration and were subjected to excitations of increasing severity, starting from very low values of accelerations. The number of three-component shocks acting on each building ranged from 3 to 7. Two types of signals were used: one about 80 secs long and the other 40 secs long. The second signal set was derived from the first one, both show basically the same frequency content (see ref.1). Table 1 lists the main characteristics of the buildings and of the relevant test sequences; figure 1 shows schematically the configuration of the buildings.

In the table, $S$ denotes the short signal and $L$ the long one, $a_{\mathrm{M}}$ is the peak acceleration in $g$ units of the last shock of the sequence. In some cases the last test was interrupted due to very heavy damage and the danger of a partial or total collapse. The latter occurred for building G1. The values of peak accelerations of interrupted shocks are reported between parentheses. Response accelerations were recorded at 18 locations during all the 58 events. Both in ref. 1 and in what follows, all results and comments are referred to the real (modeled) building.

Basing on these records absolute energies, according to the definition given in ref.2, have been computed and correlated to the observed damage. This was made for the whole set of shocks. For each of them and for each building the variation with time of the cumulated input energy $e_{I j}(t)$, where the index $j$ refers to the $j$-th shock acting on the given building, has been computed. Additionally, each base time history has been 
described by a 'referential' energy $r_{j}(t)$, defined below. Basing on these functions a 'performance' index $i_{j}(t)$ was introduced. The variations with time of $e_{I j}(t)$ and $i_{j}(t)$ are compared to the variations of the effective stiffness of the dominant mode along the considered direction.

The values of the three above functions, when computed at the end of each shock, describe respectively the total adsorbed and damping energy, the total 'referential' energy of the shock and the total 'performance' index for the given building and the given base excitation. These quantities, compared to the damage pattern detected during the relevant shock, allow to describe the global behavior of each building during the whole set of shocks that acted on it and to compare the behaviors of buildings of different type and the effects of different strengthening and repairs. These comparisons gave some hints for establishing new technical interventions on existing buildings able to enhance their energy dissipation and adsorption capacity. Moreover, the analysis of the evolution during a single shock of the energy functions derived in the next section, allows to detect the occurrence of damage, even when not fully visible. The use of these functions gives a method for the interpretation of the responses recorded during shaking table tests.

\section{ENERGY FUNCTIONS INVOLVED}

\section{a) Input, damping and absorbed energies}

Horizontal response accelerations are available, for each test, at 8 location in $x$ direction, at 8 locations in $y$ direction and at 2 locations in $z$ direction, being $x$ and $y$ the principal plan directions and $z$ the vertical one (see fig.1). In principle, the dynamic behavior of the building may be studied by associating a pertinent mass $m_{i}$ to each location, thus 
looking at the real distributed mass system as a lumped mass one. For instance, by calling $\boldsymbol{u}(t)$ and $\boldsymbol{u}_{\mathbf{t}}(t)$ the vectors of the relative and absolute displacements in $x$ direction at the said locations the following classical equation holds:

$m \ddot{u}_{t}+f_{\xi}+f_{S}=\mathbf{0}$

where $f_{S}$ is the restoring force vector, $\boldsymbol{f}_{\xi}$ is the viscous damping force vector and $\boldsymbol{m}$ the mass matrix. A similar equation may be written in $y$ direction. By integrating eq. (1) with respect to $\boldsymbol{u}$ the following energy equation in $x$ direction, at time $\mathrm{t}$, is obtained (see ref.2):

$e_{K x}(t)+e_{\xi x}(t)+e_{A x}(t)=e_{I x}(t)$

being $e_{K x}(t)$ the kinetic energy, $e_{\xi x}(t)$ the damping energy, $e_{A x}(t)$ the adsorbed energy and $e_{I x}(t)$ the input energy. The absorbed energy may be splitted into two parts:

$e_{A x}(t)=e_{H x}(t)+e_{S x}(t)$

being $e_{H x}(t)$ the hysteretic energy and $e_{S x}(t)$ the strain energy. Quantities appearing in eqs. (2) and (3) account for the responses in $x$ direction, which are also determined by the base input acting along the orthogonal direction $y$. Due to the orthogonality of inertia forces and responses in the two considered directions, the total energy equation may be written as:

$\left(e_{K x}+e_{K y}\right)+\left(e_{\xi x}+e_{\xi y}\right)+\left(e_{A x}+e_{A y}\right)=\left(e_{I x}+e_{I y}\right)$

In order to be able to derive analytically any of the above equations we should know the mechanical and dynamic properties of the system and the way they change during a given response. But we do not have this information, unless we refer to drastic simplifications and assumptions of uncertain reliability. However, basing on measured 
responses one can compute $e_{I}(t)$ and $e_{K}(t)$ and hence may know the adsorbed and damping energies $e_{A}(t)$ and $e_{\xi}(t)$, although being unable to distinguish the contributions of the different effects involved (elastic, damping and hysteretic phenomena).

At the end of the ground shaking ( $t=T$, being $T$ its duration) eqs. (2) and (3) become:

$e_{\xi x}(T)+e_{H x}(T)=e_{I x}(T)$

being $e_{K x}(T)=e_{S x}(T)=0$

In what follows quantities referred to the end of the responses will be denoted by capital letters $\left(E_{I}, E_{\xi}, E_{H}\right)$, they express the total input, damping and hysteretic energies during the given shock: at its end $E_{I}=E_{\xi}+E_{H}$.

\section{b) Examples}

As examples of some properties of the above functions, consider figures $2 a$ and $2 b$ that show the evolution of the input energy $\left(e_{I}\right)$ and of the adsorbed and damping energy $\left(e_{A}+e_{\xi}\right)$, evaluated from the responses in $x$ direction for two shocks acting on the building A1 (brick masonry, originally undamaged). The two considered shocks are respectively the first and the last one of the testing sequence for A1. The first excitations are quite modest; peak accelerations of the three components of ground motion are of the order of $5 \% \mathrm{~g}$, while the second excitations are rather severe (PGA of the order of $32 \% \mathrm{~g}$ ). During the first test the structure suffered very slight damage at spandrel beams and behaved basically linearly; during the last test the building showed heavy damage at piers and at spandrel beams of both storeys. In the two figures $e_{I}(t)$ is more jagged than $\left(e_{A}+e_{\xi}\right)$ due to the contribution of the kinetic energy to $e_{I}$. This contribution is much 
more important during the first test than during the last one, when the heavy damage suffered by the building make damping and hysteretic energies predominant with respect to $e_{K}$. If the kinetic energy $e_{K}$ is subtracted from $e_{I}$, the function $\left(e_{A}+e_{\xi}\right)$ is obtained. This function shows a similar jaggedness because of the contribution of the strain energy $e_{S}$ to $e_{A}$. This contribution is more significant during the first test (fig. 2a) than during the last one (fig. 2b), due to the intact elastic properties of the building, that progressively deteriorate at the increase of damage. During the last shock the function $\left(e_{A}+e_{\xi}\right)$ shows some steep increases, for instance at $t \cong 12,16,19 \mathrm{sec}$, which do not occur during the first test. They point out an increase of damping and hysteretic energies, connected with the occurrence of new damage or with the extension of the existing one. This feature will be further commented in the next section. The values of $e_{I}$ are quite different in the two cases, being during the last test about 105 times higher than the ones of the first test. At the end of the two events $E_{I}$ is respectively $0.232 \mathrm{kNm}$ and $24.74 \mathrm{kNm}$. Note that base inputs used in the two tests differ only in the values of accelerations, having been obtained by scaling with a constant factor the same reference signal: base accelerations of the last test are 6.4 times higher than the ones of the first test, but their frequency content is fairly similar. In the case of an ideal linear behavior, with the same dynamic properties of the original building, during the last shock one would expect energies 41 times higher than the ones of the first test. Actual values are about the double than that, as can be seen from figs $2 \mathrm{a}$ and $2 \mathrm{~b}$, thus pointing out the considerable influence of damage on energy absorption and dissipation. 


\section{c) Referential energy}

The comparison among the values of energies related to different severities of the base excitation is meaningful, as will be seen later. However, in some instances it is useful to normalize cumulated energy time histories with respect to the "importance" of the excitation.

To this purpose the following function is introduced, for each component of the ground motion:

$$
r(t)=M \int_{o}^{t}\left|\ddot{u}_{g} \dot{u}_{g}\right| d t
$$

being $M$ the total mass of the building, $u_{g}(t)$ the ground displacement acting along the considered direction. This function will be referred to as 'referential' energy. It may be seen as the work that would be made by the actuators of the table to produce the given motion $u_{g}(t)$ if the building were a rigid body of mass $M$. This function describes, although is does not measure, the energy made available to the building by the base motion $u_{g}(t)$. The total referential energy, at the end of the excitation, is denoted by $R_{x}$, $R_{y}$ or $R=R_{x}+R_{y}$ depending on the direction taken into account. Figures $3 \mathrm{a}$ and $3 \mathrm{~b}$ report $r(t)$, referred to $x$-direction, for the building A1 and for the same shocks previously cited. It may be seen that these functions are more regular than $e_{I}(t)$ and $\left(e_{A}(t)+e_{\xi}(t)\right)$ in the same direction and that they do not show steep increases like the ones of fig. $2 \mathrm{~b}$. The evolution with time of $r(t)$ is directly connected with the evolution of the base input: as a matter of example in both the considered tests the main part of the base acceleration time history (not shown here) occurs between 15 and $20 \mathrm{sec}$. and this is reflected in the correspondent increase of $r(t)$ in the same time interval (see figs. 3a 
and $3 b)$. The final values $R$ for the two tests are quite different one from the other, being the one of the last shock about 25 times larger than the one of the first test.

\section{d) Performance index}

For a better description of the behavior of a building during a given excitation and in order to allow a comparison of the structural behavior determined by shocks of different severity (which affect the values of all the above functions and parameters), the following function is introduced:

$i(t)=\frac{e_{I}(t)}{r(t)}$

$i(t)$ is called 'performance index' and is related to the same direction which $e_{I}(t)$ and $r(t)$ are referred to. Its final value $I$ may be considered as a description of the portion of the 'referential energy' provided by the ground motion that has been dissipated and adsorbed by the building during its response in the considered direction. This meaning also holds for $i(t)$ at any $t$ value. For the two tests considered up to now, $I$ is respectively 0.069 and 0.305 . The very low value for the first test is related to the total energy dissipated through viscous damping, while the higher value for the last test accounts for the important hysteretic behavior induced by the heavy damage suffered by the structure, that caused about $30 \%$ of the 'referential energy' to be dissipated and adsorbed by the building.

\section{EVOLUTION DURING A GIVEN EARTHQUAKE}

\section{a) Examples of time histories of $e_{I}, i$ and $k_{\text {eff }}$}

In all tested buildings major damage occurred in $x$-walls (with windows at both storeys and doors at the first one). For this reason reference is made here to energy and index 
time histories evaluated from the signals recorded along $x$-direction, although these functions have been determined also along the orthogonal $y$-direction. In the following energy notations the suffix $x$ is dropped. In order to clarify the main items that characterize the response in terms of the above functions, in this section reference is made to the systems $\mathrm{A} 1$ and $\mathrm{C} 1$.

Figures $4 \mathrm{a}$ and $4 \mathrm{~b}$ show respectively, for the first and last shock on $\mathrm{A} 1$, the functions $\left(e_{A}+e_{\xi}\right), i(t), r(t)$ and the effective stiffness $k_{\text {eff }}$ of the first mode, which turned out to be predominant during the response. The effective stiffness $k_{\text {eff }}$ is defined (see ref.1) as the ratio of the restoring forces to the relative displacement pertaining to the first mode. It is computed by band-pass filtering responses at a given location, around the modal frequency, previously determined through system identification. Modal restoring forces and displacements are correlated by quasi-elliptic cycles: the slope of their major axes determines the effective stiffness of the time corresponding to the maximum cycle displacement. In the case of perfectly linear response, the slope of the cycles is unchanged during the response. When the structure deteriorates cycles widen and their slope decreases. The analysis of the evolution of $k_{\text {eff }}$ during the base motion allows to detect the occurrence of damage, marked by a decrease of $k_{e f f}$.

\section{b) Some properties of $e_{I}$, $i$ and $k_{\text {eff }}$}

Cumulative energy functions are normalized to their peak values in order to facilitate their comparison. The final values are, infact very different one from the other: for the first excitation $E_{I}=0.23 \mathrm{kNm}, R=3.3 \mathrm{kNm}$ and for the last one $E_{I}=24.74 \mathrm{kNm}, R=81$ $\mathrm{kNm}$. During the first excitation the building has a basically linear behavior (with only very modest damage at spandrel beams) with a significant contribution of the strain 
energy to $e_{A}$. Thus the maximum values of $\left(e_{A}+e_{\xi}\right)$ occur during the shock, being the final input energy $E_{I}$ a little lower than it (fig. 4a). This feature does not occur when the building is heavily damaged (fig.4b), in these instances maximum values of $\left(e_{A}+e_{\xi}\right)$ are recorded at the end of the excitation. In both cases $r(t)$ is relatively regular, with only some limited increases (e.g. at $t=35 \mathrm{sec}$. for the first test and at $t=33 \mathrm{sec}$. for the last one and between 15-20 sec. for both) which are due to the correspondent increase of base accelerations. When the system is practically undamaged (fig. 4a) the effective stiffness $k_{\text {eff }}$ is fairly constant during the response and the function $\left(e_{A}+e_{\xi}\right)$, although very jagged due to the influence of the strain energy, does not show steep increases in its mean value. On the contrary, during the last base motion, when the building shows an increasing heavy damaged state, the function $\left(e_{A}+e_{\xi}\right)$ has some steep increases occurring at $t=12.8,16.4,19.8,24 \mathrm{sec}$. For this shock the effective stiffness starts to decrease a little before the beginning of the steep increase of the input energy. The reduction of the stiffness is a signal of new damage on the building or of the extension of the existing one: the effect is the increase of damping and absorbed energy which is reflected on the evolution of $\left(e_{A}+e_{\xi}\right)$. In correspondence to the time at which $k_{\text {eff }}$ starts to decrease, the referential energy $r(t)$ increases more rapidly than it made in the previous time intervals and base accelerations become more significant: this is made apparent either by an augmented slope of the curve $r(t)$ or by its relatively sharp increase.

The increased severity of the base motion affects the amount of the absorbed and damping energy after a delay $\Delta t$, of the order of 1-2 sec: it follows that the function $\left(e_{A}+e_{\xi}\right)$ varies with a minor slope than $r(t)$ during $\Delta t$. According to the way $i(t)$ is defined, it decreases in $\Delta t$, being $r(t)$ at the denominator. In turn, when damage affects 
the energy dissipation and absorption capacity of the building, producing its growth, $i(t)$ increases following the increase of $\left(e_{A}+e_{\xi}\right)$. The occurrence of damage is thus characterized by valleys in the function $i(t)$.

\section{c) Qualitative evolution and correlation to damage}

The analysis of the evolutions with time of $r(t),\left(e_{A}+e_{\xi}\right), i(t), k_{\text {eff }}$ related to all the damaging shocks acting on the buildings of Table 1, pointed out that damage is marked by the items above described: $(\boldsymbol{a})$ reduction of $k_{\text {eff }},(\boldsymbol{b})$ concurrent augmented slope of $r(t)$; $(\boldsymbol{c})$ valleys of $i(t) ;(\boldsymbol{d})$ sharp increases of $\left(e_{A}+e_{\xi}\right)$, which occurs slightly after the beginning of phase $(\boldsymbol{a})$.

These steps are qualitatively shown in fig.5; at time $t_{\mathrm{a}} r(t)$ increases its slope and $i(t)$ starts to decrease, the same happens with $k_{\text {eff. }}$ The effect on $\left(e_{A}+e_{\xi}\right)$ is felt a little after, at time $t_{\mathrm{b}}$, when $i(t)$ again increases. After $t_{c}$ a recover of stiffness occurs and the variation of $\left(e_{A}+e_{\xi}\right)$ is slower. Valleys of $i(t)$ and steep changes of $\left(e_{A}+e_{\xi}\right)$ mark the occurrence of damage but do not enable to distinguish its nature (e.g. to spandrel beams or to piers). Damage is, infact, detected after the shock and the final value of input energy may be only attributed to variation of the damage pattern with respect to the previous excitations. The only exceptions are when buildings suffer only damage of a given type during the first excitation: in this case it is possible to assess the amount of energy dissipation and adsorption connected to it. As an example, fig.4a shows a damage occurring at $t=14.7 \mathrm{sec}$. : the detected damage after the shock was a very slight one at spandrel beams; it caused a sudden variation of absorbed energy of about $12 \%$ the final one. The effect of damage is, however, felt also after the time interval at which it occurred. The increase of damping, produced by it, is reflected in the increase of the 
slope of $\left(e_{A}+e \xi\right)$ after $t=14.7 \mathrm{sec}$. The important role played by damage to spandrel beams on energy dissipation and absorption capacities of the buildings is confirmed by the analysis of all the cases where it occurred. This item will be again commented in the next section. During the first part of the excitations $i(t)$ tends to decrease with respect to the initial values. For buildings responding basically in a linear way (as the case of fig.4a) this is due to the predominance of the strain energy on the cumulated damping and adsorbed energy. This causes an average slope of $\left(e_{A}+e_{\xi}\right)$ lower than the corresponding slope of $r(t)$ (see fig.4a in the time interval 15-20 sec).

\section{d) Effects of the vertical component of motion}

For events causing important damage, the initial decrease of $i(t)$ may be due also to another reason. As an example, consider fig. 6 referred to the last shock on building $\mathrm{C} 1$ (before the interrupted one) and to quantities evaluated along $x$-direction. $\mathrm{C} 1$ is a brick masonry building: it was tested by a series of base inputs of long duration, in the figure energies are normalized to their maximum values. Figure 7 shows the initial portions of the base inputs in $x$ direction and the vertical one for the said event. Peak accelerations are about $0.26 \mathrm{~g}$ in both cases. The final values of the input and 'referential' energies are respectively $17.5 \mathrm{kNm}$ and $105 \mathrm{kNm}$. During the base motion the structure suffered severe damage at spandrel beams of the two storeys. The evolutions of $\left(e_{A}+e_{\xi}\right)$, of $i(t)$ and of $k_{\text {eff }}$ suggest that damage or its extension occur at times $t=14,17,21$ and $51 \mathrm{sec}$. (fig. 6). The stiffness decrease starts to appear at $t=11.8 \mathrm{sec}$. Until then, the values of horizontal accelerations are rather low, of the order of $0.04 \mathrm{~g}$ while the vertical excitation is considerably higher, being of the order up to $0.18 \mathrm{~g}$. Additionally, the Fourier analysis of the two signals shows a richer high frequencies content for the vertical input than for 
the horizontal one. The decrease of $k_{\text {eff }}$ cannot be attributed to horizontal actions, due to their very low values up to the considered time. A possible interpretation is that the high frequency components (of relatively high amplitudes) of the vertical signal cause the deterioration of the mechanical properties of the mortar, what is reflected in the decrease of $k_{\text {eff. }}$. The subsequent increasing horizontal accelerations act on a structure which already lost the properties it had at the beginning of the shock and hence produce damage. This feature was observed in all buildings tested in their original configuration, but was not in repaired ones. Infact, repairs did not affect the quality and the nature of the mortar, that was damaged after the test sequence related to the buildings in their original configuration.

The shaking table tests referred to in this paper gave some hints of the influence of the generally neglected vertical components of motion; this influence deserves further investigations.

\section{CORRELATION BETWEEN DAMAGE AND TOTAL ENERGIES}

\section{a) Correlation among $E, R$ and $I$}

The final values of referential and input energies are useful tools in order both to describe the behavior of a single building during the whole sequence of excitations acting on it and to compare the global behavior of different buildings.

To this aim we can make reference either to quantities evaluated in a given direction (such as $E_{I x}, E_{I y}, R_{x}, R_{y}$ ) or to total quantities (e.g. $E_{I t}=E_{I x}+E_{I y}$ ). Since two sets of excitations of different length were used (see Table 1) and energies involved depend on the duration of the signals, energies pertaining to buildings subjected to longer shocks 
(B1, B2, C1, C2) were reduced by the ratio of the time length of the shorter shocks (approx. $40 \mathrm{sec}$. ) to the one of the longer duration (approx. $80 \mathrm{sec}$. )

As an example of the information that may be derived by the evolution of final input energies during the whole set of excitations acting on a given building, consider fig.8, which refers to the system B1 and to quantities computed along $x$ and $y$ directions. Input energies $E_{I x}$ and $E_{I y}$ are plotted against 'referential' energies $R_{x}$ and $R_{y}$. For the last two shocks, base inputs acting along $x$ and $y$ are rather different, this is reflected in the different values of their $R_{x}$ and $R_{y}$. The figure points out the diversity of the response of the building along the two considered directions, which depends on the different energy absorption and dissipation capacities of $x$-walls and $y$-walls. During the first two shocks no damage was recorded, some very small cracks were detected at spandrel beams of $x$ walls after the third test. This damage became significant during the fourth and the last excitations; $y$-walls showed some damage only during the last shock. The evolution of the damaged pattern corresponds to the values and to the variations of $E_{I x}$ and $E_{I y}$, being $E_{I x}$ always considerably greater than $E_{I y}$ and the slopes of $E_{I x}-R_{x}$ curve greater than the ones of $E_{I y}-R_{y}$ curve. This fact indicates the significant effect of damage to spandrel beams on energy dissipation and absorption. The fourth event marks an important change of the response; this is also described by the change of modal parameters occurring after it and reported in ref.1. This event, denoted by $P_{y}$, may be considered as the one producing the 'significant yield' in the structure; as a matter of fact, lateral force-displacement curves worked out in ref.1 indicate $P_{y}$ as the end of the 'linearizable behavior', in agreement with what is derived from the present energy analysis. It may be noted that the slope of the $(E, R)$ curves is fairly constant up to the event $P_{y}$. This is reflected in the evolution of the performance indexes $I_{x}$ and $I_{y}$ against $R_{x}$ and $R_{y}$, shown 
in fig.9. Such indexes are rather constant up to $P_{y}$. This feature is recovered for all the tested buildings: significant yield marks the end of the quasi-constant trend of the index. The quasi-constant evolution of $I_{x}$ and $I_{y}$ up to $P_{y}$ is not surprising. Infact, tests have been carried out by scaling base inputs of a factor $\gamma>1$ with respect to the reference signals. In the ideal case of perfect linear responses both $E$ and $R$ change of $\gamma^{2}$ and hence $I \sim$ cost. The very modest deviations from $I \sim \operatorname{cost}$ detected in the tested buildings may be attributed to increases of damping due to slight, and not visible, damage to the mortar. After $P_{y}$, when serious damage occurs, important hysteretic effects appear in the structure and $E_{I}$ increases more rapidly than $R$, both in $x$ and $y$ direction thus producing the change of $I$. Figure 9 shows that $I_{x}$ is always higher than $I_{y}$, as a consequence both of the larger energy absorption and dissipation capacity of $x$-walls and of the more significant damage suffered by them. The performance index may hence be assumed also to describe the amount of damage to a given structure and its distribution among the main resisting elements ( $x$ and $y$ walls).

\section{b) Significant phases of behavior}

Figures like 8 and 9 summarize the 'history' of a given building through the sequence of excitations acting on it, they have been worked out for all the tested systems. Three points are of interest, in what they describe three different phases of behavior: the first one $P_{i}$ is related to the basically linear response to the first shock, with peak base inputs of the order of $0.05 \mathrm{~g}$, the second one $P_{y}$ refers to the significant yield, the third point $P_{u}$ refers to the response to the last shock. In most instances, particularly for repaired and strengthened systems, the last test caused very severe damage and the corresponding structural behavior may be considered as the ultimate one. For brick masonry buildings, 
tested in their original configuration (A1, B1, C1, D1), damage produced by the last shock was heavy but not so severe as to consider the structure in its ultimate state, $P_{u}$ denotes hence situations close to it. For most stone masonry buildings the last test was interrupted to avoid the total collapse, which however occurred for the system G1.

Table 2 shows the final values of the 'performance indexes' $I_{x}, I_{y}$, and $I_{t}$ evaluated at the three considered phases of behavior $P_{i}, P_{y}$ and $P_{u}$. Values referred to interrupted tests are between parentheses. The comparison between $I_{x}$ and $I_{y}$ gives some information about the different engagement, at $P_{i}$, and about the different damage, at $P_{y}$ and $P_{u}$, of $x$ and $y$ walls. In most cases $x$-walls are more engaged (and damaged) than $y$-walls. Only when a r.c. band is applied at the two story levels as it happened for building E1 (see fig.10), $I_{y}>I_{x}$. In these cases, spandrel beams are much stronger and stiffer than in the other configurations and hence reduce the energy dissipation and absorption capacity of $x$-walls.

The values of $I_{t}$ at $P_{i}$ are a description of the initial quality of the buildings before the sequence of excitations acting on them. Original BM systems are rather homogeneous, with $I \sim 0.07$; the only exception is D1 which proved to be much better constructed than the other BM systems, showing $I \sim 0.018$.

Among SM buildings, G1 and F1 have initially higher indexes than E1 and H1.This is a consequence of the very poor quality of construction for G1, which infact collapsed during a relatively moderate shock, and of damage caused on F1 during the transportation to the shaking table. It may be noted that the index is able to account for these mishaps and might be used for diagnostic purposes of real buildings, provided responses, even to very low base inputs, are available. 


\section{c) Effects of retrofittings on $I_{t}$ and $E_{I t}$}

It may be seen from Table 2 that for the original BM buildings $I_{x}>I_{y}$ at all the three considered phases of behavior, while after repairs this was the case only up to the significant yield $P_{y}$, being at ultimate $I_{x} \leq I_{y}$. These facts may be explained by considering that repaired $\mathrm{BM}$ buildings were subjected to final shocks that were considerably more severe than those acting on the original configurations, hence their behavior may be properly considered as the ultimate one. Additionally, all repairs and strengthenings were aimed at connecting all the walls of the building (see Table 1) by means of horizontal tendons or internal and external r.c. bands. These proved able to fully engage, during the final response, both $x$ and $y$ walls, as a consequence of a 'compact' box-type response mechanism. The same holds for the two SM buildings E1 and F1 strengthened in a similar way. It turns out that those interventions enforce a more homogeneous global behavior at ultimate of buildings than the other considered ones.

Figures 11, 12 and 13 show $E_{I t}$ as a function of $R_{t}$ for all the tests carried out respectively on BM buildings in their original (fig.11) and repaired (fig.12) configurations and on SM systems (fig.13). It may be seen again that buildings subjected to prior $(\mathrm{SM})$ or repair $(\mathrm{BM})$ interventions show a reduced scatter with respect to $\mathrm{BM}$ original systems, thus supporting the above comments about the effect of repairs and strengthenings. Maximum values of $R_{t}$ and $E_{I t}$ occur for repaired BM buildings: they are respectively 7 and 6.5 times higher than the corresponding largest ones of SM buildings due to the larger base excitations needed to cause ultimate conditions on strengthened BM systems. During the initial phase of behavior and up to $P_{y}$, repaired BM buildings engage energy dissipation capacities higher than the ones of the corresponding original systems: this may be seen from the values of $I_{t}$ at $P_{i}$ and $P_{y}$ reported in Table 2. Stone 
masonry buildings, in turn, have values of the total 'performance' index during the same phases which are $2 \div 4$ times higher than the ones determined for BM systems. Up to the significant yield input energy is mainly due to non-hysteretic phenomena, being damage still limited. At $P_{y}$ equal values of the referential energy $R_{t}$ correspond to total input energies $E_{I t}$ that are $2 \div 4$ times higher for SM systems than for BM ones: stone masonry dissipates significantly more than brick masonry. This result is consistent with the values of damping identified in ref. 1 for SM and BM up to $P_{y}$.

However, during very severe excitations, engaging buildings to their ultimate states and causing heavy damage, $I_{t}$ values for SM systems and for strengthened BM ones are very similar (of the order of 0.4), although the total absorbed and damping energies $E_{I t}$ are higher for strengthened BM buildings than for SM ones, due to the longer base inputs involved in the final responses of BM structures. This fact shows that the hysteretic behavior occurring at ultimate is similar in the two types of buildings, and is independent on the material employed for construction.

If 'anomalous' buildings (D1, F1 and G1) are excluded (D1 was of much better quality than the other BM systems, F1 was damaged during the transportation to the shaking table and G1 was very poorly built, with practically no connections between orthogonal walls), Table 2 shows that at significant yield $I_{t}$ is around $0.06-0.1$ for BM systems and 0.1-0.15 for SM ones; at ultimate $I_{t}$ is about $0.30-0.35$ for BM (if only true ultimate conditions are taken into account) and about $0.34-0.38$ for SM buildings. The above values roughly characterize different phases of behavior of masonry buildings: a their possible use is the tentative assessment of the 'position' relative to $P_{y}$ and $P_{u}$ of a given building for which base and response signals are available. The comparison to them of its actual I provides such rough assessment. 


\section{d) Preferable damage states}

After each excitation buildings have been surveyed in order to assess the evolution of the damage pattern. The type of damage (new one or extension of the existing one) recorded between two shocks was correlated to the relevant variations $\Delta R$ and $\Delta E_{I}$ of referential and input energies. A detailed description of these correlations is not given here due to space limitations. We only draw the attention on one of the most important phenomena that have been observed and previously mentioned: the effect of damage to spandrel beams on energy absorption. To this aim consider first fig. 14 where $E_{I x}$ is plotted against $R_{x}$ for all the shocks acting on building A2. This structure was repaired by the application of r.c. bands at each level, similarly to E1: their effect was to tie efficiently together the building and to strengthen significantly spandrel beams, which infact did not suffer important damage up to the very last excitation, during which important damage was recorded at spandrel beams. The last base motion was practically identical to the previous one, as shown by the very similar values of $R_{5 x}$ and $R_{6 x}$. Correspondingly, input energies between test 5 and test 6 vary of about $40 \%$. The variation of damage between the two tests consists mainly in very serious cracks appearing at spandrel beams in $x$-walls. It turns out that the increase of input energy has to be attributed to damage to spandrel beams. In order to make this point clearer it is convenient to introduce the following indexes:

$$
\alpha_{R}=\frac{\Delta R}{R_{1}} \quad \alpha_{E}=\frac{\Delta E_{I}}{E_{1}} \quad \alpha=\frac{\alpha_{E}}{\alpha_{R}}
$$

being $\Delta R$ and $\Delta E_{I}$ the increments of referential and input energies in a given direction occurring between two subsequent shocks and $R_{l}$ and $E_{l}$ the energies related to the first of the two considered excitations. 
In the case of perfectly linear behavior $\alpha=1$; when $\alpha>1$ the greater $\alpha$ the greater nonlinear behavior occurs, with energy absorption and dissipation capacity increasing more than the base referential energy. In some buildings, damage occurred during the last two shocks only at spandrel beams, predominantly of $x$-walls. This happened for buildings B1, C1, D1. On the other hand for building E1, which was strengthened by using r.c. bands at each level, damage was concentrated at piers, with only some small cracks at spandrel beams. By computing $\alpha$ with reference to $x$-direction and to the last two shocks, the following values were obtained: $\alpha=3.45,3.98,5.16$ for B1, C1 and D1; $\alpha=2.57$ for E1. For E1 the same value of $\alpha$ is also found if the third and fourth shocks are considered, that is when important damage occurs first at piers. The above values quantify the greater influence on energy absorption and dissipation due to damage to spandrel beams with respect to the one to piers. For the same increments of referential energy, which is a description of the severity of the shock, damage to spandrel beams involves increments of input energy that are $40-100 \%$ higher than the ones related to pier cracking. This result may influence the choice of the repair/strengthening interventions on masonry buildings, promoting the study of devices that allow the highly energy consuming damage to spandrel beams, although controlling it. This type of damage is more preferable than the one to piers: their collapse determines infact the collapse of the entire structure.

\section{CONCLUSIONS}

Energy functions have been evaluated from the responses to the 58 base excitations acting on the 12 stone and brick masonry buildings. The severity of each shock is described by $r(t)$, depending on the considered ground motion, while the behavior of the 
building during the excitation is described by $i(t)$, the ratio to $r(t)$ of the input energy $e_{I}(t)$. The function $i(t)$ was found to be a good descriptor of the behavior of the structure: when the structure responds linearly, $i(t)$ is basically constant; valleys in $i(t)$ correspond to sharp increases of $e(t)$ and denote the occurrence or the extension of damage. The variations of the performance index with time are consistent with the variations of modal parameters of the dominant mode during the response, as was mentioned. The analysis of the above functions enables to detect the occurrence of damage during the response and may be seen as a method, based on energy functions, for the interpretation of seismic responses. Its application provided some hints for assessing the effect of the vertical component of ground motion on the structural behavior.

The comparison of $R, I$ and $E_{I}$ (the values of the above functions at the end of the excitation) pertaining to the considered shocks and buildings allows a quantitative description of the energy dissipation and absorption capacities connected with the different materials of the buildings, with their state of damage and with the different strengthening considered. Of particular interest is the influence of damage to spandrel beams on the energy absorption capacity, which is much higher than the one determined by other types of damage. This fact may result into a new strengthening strategy, aimed at allowing the occurring of this damage, though controlling it, in order to protect from collapse the structure. This item may be considered as new, for the type of buildings herein considered, but needs further analysis. 
e) References

1. D. Benedetti, P. Carydis, P. Pezzoli, 'Shaking table tests on 24 masonry buildings', Earth. Eng. struct. dyn. 1998; 27: 67-90.

2. C. M. Uang and V.V. Bertero, Evaluation of seismic energy in structures, Earth. Eng. struct. dyn. 1990; 19: 77-90. 
Table 1

\begin{tabular}{|c|c|c|c|c|c|}
\hline Building & Mat. & $\begin{array}{c}\text { Repair/ } \\
\text { Strengthening }\end{array}$ & $\begin{array}{c}\text { aM } \\
(\mathbf{g} \text { units })\end{array}$ & Input & $\begin{array}{c}\text { N. of } \\
\text { shocks }\end{array}$ \\
\hline A1 & BM & $s c$ & 0.32 & S & 5 \\
\hline A2 & BM & $l s c-r b-s n 2$ & 0.43 & S & 6 \\
\hline B1 & BM & --- & 0.33 & L & 5 \\
\hline B2 & BM & $l s c-s n 1,2$ & 0.42 & $\mathrm{~L}$ & 5 \\
\hline C1 & BM & --- & $0.26(0.27)$ & $\mathrm{L}$ & 4 \\
\hline C2 & BM & $l s c-h t-s n 1,2$ & 0.44 & $\mathrm{~L}$ & 5 \\
\hline D1 & BM & $s c$ & 0.30 & S & 5 \\
\hline D2 & BM & $l s c-h t$ & 0.32 & S & 7 \\
\hline E1 & SM & $s c-r b$ & $0.23(0.24)$ & S & 5 \\
\hline F1 & SM & $s n l, 2$ & $0.16(0.17)$ & S & 4 \\
\hline G1 & SM & --- & $0.17(0.19)$ & S & 4 \\
\hline H1 & SM & $s c-h t$ & 0.16 & S & 3 \\
\hline
\end{tabular}

$(B M)$ brick masonry, $(S M)$ roughly squared stone masonry, $(s c)$ steel connectors, $(l s c)$ local sealing using cement mixture, $(s n i)$ steel network on slabs ( $i$ denotes the storey level), $(r b)$ reinforced concrete band, $(h t)$ horizontal tendons.

Table 2

\begin{tabular}{|c|c|c|c|c|c|c|c|c|c|}
\hline Model & \multicolumn{3}{|c|}{$\boldsymbol{P}_{i}$} & \multicolumn{3}{|c|}{$\boldsymbol{P}_{y}$} & \multicolumn{3}{|c|}{$\boldsymbol{P}_{u}$} \\
\hline & $I_{x}$ & $I_{y}$ & $I_{t}$ & $I_{x}$ & $I_{y}$ & $I_{t}$ & $I_{x}$ & $I_{y}$ & $I_{t}$ \\
\hline f) $A 1$ & 0.069 & 0.028 & 0.047 & 0.123 & 0.042 & 0.08 & 0.305 & 0.278 & 0.291 \\
\hline A2 & 0.09 & 0.0628 & 0.075 & 0.1156 & 0.0957 & 0.1045 & 0.249 & 0.45 & 0.38 \\
\hline B1 & 0.077 & 0.012 & 0.045 & 0.091 & 0.018 & 0.049 & 0.179 & 0.037 & 0.099 \\
\hline B2 & 0.13 & 0.034 & 0.079 & 0.121 & 0.037 & 0.077 & 0.25 & 0.313 & 0.282 \\
\hline $\mathrm{C1}$ & 0.062 & 0.023 & 0.041 & 0.092 & 0.035 & 0.061 & $\begin{array}{l}0.17 \\
(0.42)\end{array}$ & $\begin{array}{l}0.09 \\
(0.30)\end{array}$ & $\begin{array}{l}0.12 \\
(0.35)\end{array}$ \\
\hline $\mathrm{C} 2$ & 0.101 & 0.013 & 0.054 & 0.111 & 0.023 & 0.065 & 0.215 & 0.34 & 0.274 \\
\hline D1 & 0.018 & 0.015 & 0.017 & 0.021 & 0.03 & 0.025 & 0.092 & 0.062 & 0.076 \\
\hline D2 & 0.067 & 0.065 & 0.066 & 0.132 & 0.06 & 0.091 & 0.198 & 0.24 & 0.22 \\
\hline E1 & 0.062 & 0.108 & 0.087 & 0.086 & 0.128 & 0.108 & $\begin{array}{l}0.19 \\
(0.44)\end{array}$ & \begin{tabular}{|l|}
0.18 \\
$(0.365)$ \\
\end{tabular} & $\begin{array}{l}0.19 \\
(0.406) \\
\end{array}$ \\
\hline F1 & 0.197 & 0.213 & 0.206 & 0.212 & 0.20 & 0.20 & $\begin{array}{l}0.27 \\
(0.388)\end{array}$ & \begin{tabular}{|l|}
0.23 \\
$(0.379)$ \\
\end{tabular} & \begin{tabular}{|l}
0.24 \\
$(0.384)$ \\
\end{tabular} \\
\hline G1 & 0.246 & 0.23 & 0.236 & 0.335 & 0.24 & 0.284 & 0.33 & 0.23 & 0.25 \\
\hline H1 & 0.121 & 0.098 & 0.109 & 0.146 & 0.144 & 0.145 & 0.45 & 0.253 & 0.345 \\
\hline
\end{tabular}




\section{Titles of tables}

Table 1. Tested buildings

Table 2. Values of $I$ at the three phases of behavior of all the tested buildings. 


\section{Captions of figures}

1. Scheme of the tested buildings (real sizes).

2a. Energies evaluated from the responses in $x$-direction for the first test ( $a_{M} \cong 0.05 \mathrm{~g}$ )on building A1.

2b. Energies evaluated from the responses in $x$-direction for the last test ( $a_{M} \cong 0.32 \mathrm{~g}$ ) on building A1.

3a. Referential energy in $x$-direction for the first shock on building A1

3b. Referential energy in $x$-direction for the last shock on building A1

4a. Absorbed and damping energy, referential energy, performance index and effective stiffness of the first mode evaluated from responses in $x$-direction during the first shock $\left(a_{M} \cong 0.05 \mathrm{~g}\right)$ on building $\mathrm{A} 1$. Energies are normalized to their maximum values.

4b. Absorbed and damping energy, referential energy, performance index and effective stiffness of the first mode evaluated from responses in $x$-direction during the last shock $\left(a_{M} \cong 0.32 \mathrm{~g}\right)$ on building $\mathrm{A} 1$. Energies are normalized to their maximum values.

5. Qualitative evolution of $i, e, r$ and $k_{\text {eff }}$ at the occurrence of damage.

6. Adsorbed and damping energy, referential energy, performance index and effective stiffness of the first mode evaluated from responses in $x$-direction during the last shock $\left(a_{M} \cong 0.27 \mathrm{~g}\right)$ on building $\mathrm{C} 1$. Energies are normalized to their maximum values.

7. Initial portions of the horizontal (in $x$-direction) and vertical acceleration acting on C1 (last test). 
8. Input energies vs. referential energies computed for all the shocks acting on building B1. Numbers 1-5 denote the shocks of the testing sequence.

9. Performance index vs. referential energies for building B1.

10. Building E1 strengthened by r.c. bands.

11. Input energy vs. referential energy for original BM systems.

12. Input energy vs. referential energy for repaired BM systems.

13. Input energy vs. referential energy for SM systems.

14. Input and referential energies in $x$-direction for building A2. 